\title{
Design and Analysis for a 60-GHz Low-Noise Amplifier With RF ESD Protection
}

\author{
Bo-Jr Huang, Student Member, IEEE, Chi-Hsueh Wang, Member, IEEE, Chung-Chun Chen, Student Member, IEEE, \\ Ming-Fong Lei, Member, IEEE, Pin-Cheng Huang, Student Member, IEEE, \\ Kun-You Lin, Member, IEEE, and Huei Wang, Fellow, IEEE
}

\begin{abstract}
An RF electrostatic discharge (ESD) protection for millimeter-wave (MMW) regime applied to a $60-\mathrm{GHz}$ low-noise amplifier (LNA) in mixed-signal and RF purpose 0.13- $\mu \mathrm{m}$ CMOS technology is demonstrated in this paper. The measured results show that this chip achieves a small signal gain of $20.4 \mathrm{~dB}$ and a noise figure (NF) of $8.7 \mathrm{~dB}$ at $60 \mathrm{GHz}$ with $65-\mathrm{mW}$ dc power consumption. Without ESD protection, the LNA exhibits a gain of $20.2 \mathrm{~dB}$ and an NF of $7.2 \mathrm{~dB}$ at $60 \mathrm{GHz}$. This ESD protection using an impedance isolation method to minimize the $\mathrm{RF}$ performance degradation sustains $6.5-\mathrm{kV}$ voltage level of the human body model on the diode and $1.5 \mathrm{kV}$ on the core circuit, which is much higher than that without ESD protection $(<350 \mathrm{~V})$. To our knowledge, this is the first CMOS LNA with RF ESD protection in the MMW regime and has the highest operation frequency reported to date.
\end{abstract}

Index Terms-CMOS integrated circuits (ICs), low-noise amplifiers (LNAs), millimeter-wave (MMW) circuits, RF electrostatic discharge (ESD) protection.

\section{INTRODUCTION}

$\mathbf{E}$ LECTROSTATIC discharge (ESD) protection has always been a great concern in low voltage deep-submicrometer CMOS technology. The reliability issue in CMOS integrated circuits (ICs) due to smaller feature sizes becomes more and more critical. With thinner gate oxide thickness, CMOS circuits are more sensitive to the stress from ESD. During manufacture and assembly of ICs, ESD may cause IC damage. Despite the mature technology of high speed switching and high-voltage robustness for dc ESD protection [1], RF ESD protection design in state-of-the-art CMOS technologies is still a challenge, not to say in the millimeter-wave (MMW) region. A major difficulty is the large parasitic capacitances of the ESD protection devices. The conventional ESD protection is shown in Fig. 1. A power clamp between $V_{D D}$ and ground is used for dc ESD protection, while a pair of bi-directional diodes is directly added to the input port for RF ESD protection [2]. However, as the frequency increases to gigahertz frequency range, the parasitic capacitance

Manuscript received January 30, 2008; revised October 01, 2008. First published January 06, 2009; current version published February 06, 2009. This work was supported by the National Science Council of Taiwan and Excellent Research Projects of National Taiwan University under Grant NSC NSC 2221-E-002-144 and 97R0062-03.

The authors are with the Department of Electrical Engineering and Graduate Institute of Communication Engineering and National Taiwan University, Taipei 10617, Taiwan (e-mail: kunyou@cc.ee.ntu.edu.tw; hueiwang@ew.ee.ntu.edu. tw).

Color versions of one or more of the figures in this paper are available online at http://ieeexplore.ieee.org.

Digital Object Identifier 10.1109/TMTT.2008.2011158

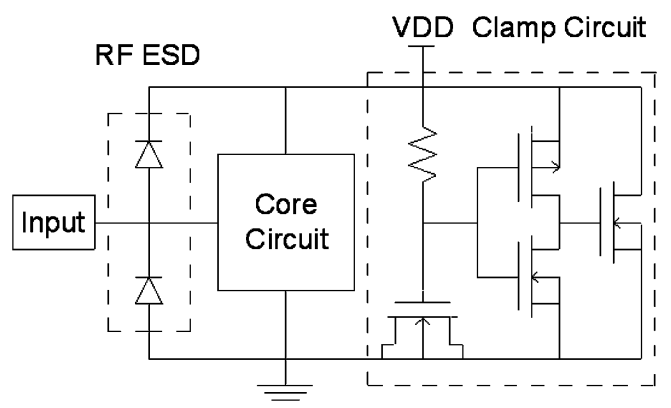

Fig. 1. Conventional ESD protection scheme with a power clamp.

at such a high frequency acts as a short path and will seriously degrade the performance of the core circuit due to the mismatch to RF networks.

ESD robustness is usually proportional to the device size, i.e., the higher parasitic capacitance, the more robust the ESD is. Therefore, there is a tradeoff between ESD robustness and RF performance, especially over wide bandwidth.

In RF systems, such as a heterodyne receiver, the LNA is a critical building block in the front-end. This is because it is usually connected to outside through the antenna or an off-chip antenna filter and can be easily exposed to the ESD stress events. Thus, it is important to incorporate the RF ESD protection in an LNA.

In this paper, a structure of impedance isolation method for RF ESD protection applied to a $60 \mathrm{GHz}$ LNA in $0.13-\mu \mathrm{m}$ CMOS technology is demonstrated and analyzed. The LNAs, with and without ESD protection, are designed and fabricated and the performances such as gains, return losses, noise figures (NFs), etc. are compared. Without ESD protection, the LNA achieves a maximum small-signal gain of $20.2 \mathrm{~dB}$ and a minimum NF of $7.2 \mathrm{~dB}$ from 55 to $63 \mathrm{GHz}$. The ESD-protected LNA shows a 20.4-dB gain and an 8.7-dB NF. The gain variation is very small and the NF variation is $1.5 \mathrm{~dB}$ between the LNAs with and without ESD protection. The diodes failed under more than $6.5-\mathrm{kV}$ human body model (HBM) testing and the core circuit can still retain its performance under $1.5-\mathrm{kV}$ testing.

\section{Circuit Implementation}

\section{A. LNA Design}

The LNA is designed based on TSMC commercial $0.13-\mu \mathrm{m}$ mixed-signal/RF (MS/RF) purpose CMOS process, which provides one poly layer for the gates of CMOS transistors and eight metal layers for inter-connection (1P8M) with an ultra-thick top 


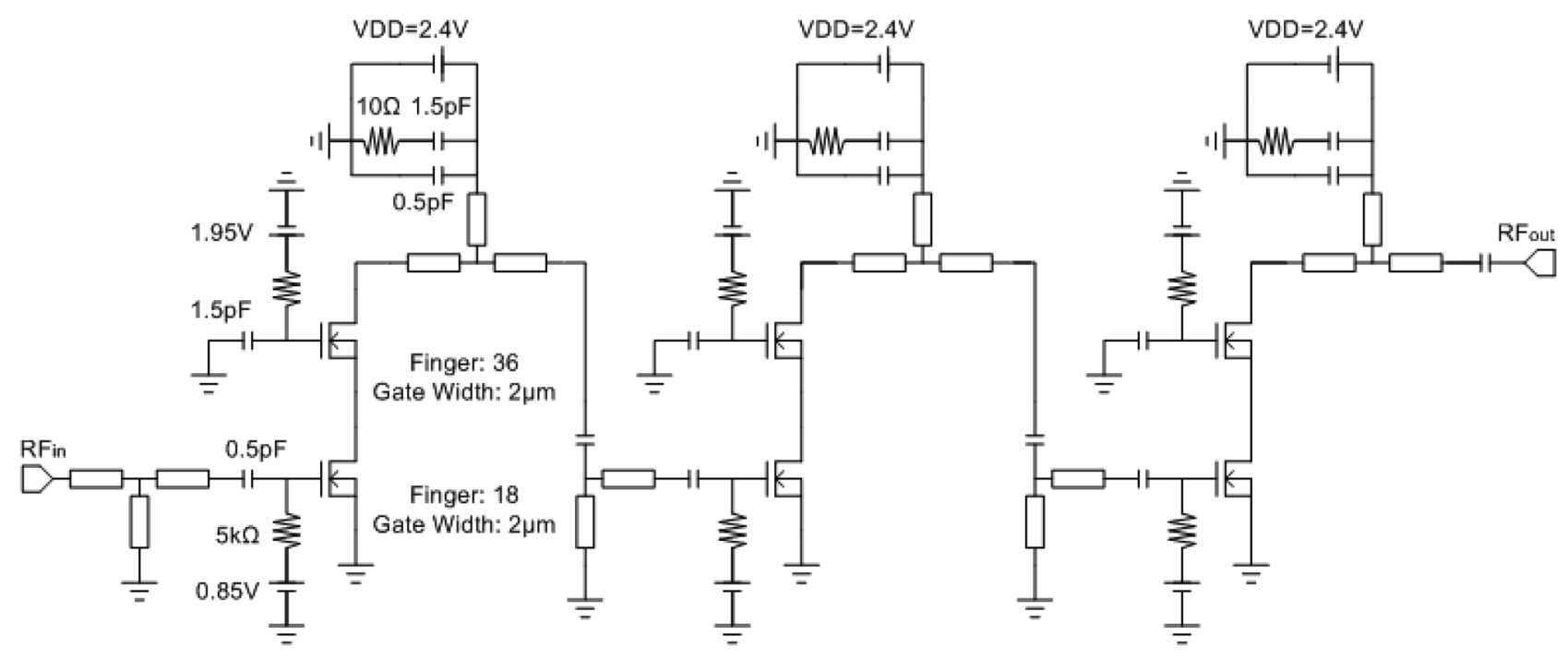

Fig. 2. Three-stage schematic of the proposed $V$-band LNA.

metal of 3.3- $\mu \mathrm{m}$. The metal-insulator-metal (MIM) capacitors and polysilicon resistors are available. The CMOS process features $f_{\max }$ and $f_{t}$ of 108 and $91 \mathrm{GHz}$ with a $1.2-\mathrm{V}$ supply, respectively [3].

In the LNA design, the cascode configuration [3] is utilized to achieve high gain performance. It is known that different device size combinations in a cascode configuration exhibit various maximum stable power gains (MSGs). As discussed in [3], the device sizes are selected for the highest MSG at $60 \mathrm{GHz}$. The size of the common-source and common-gate NMOS transistors are 18-finger with total gatewidth of $36 \mu \mathrm{m}$, and 36-finger with total gatewidth of $72 \mu \mathrm{m}$, respectively. Under a class-A bias condition, the cascode configuration exhibits a maximum available power gain of $11.5 \mathrm{~dB}$, and a minimum NF of $5 \mathrm{~dB}$ at $60 \mathrm{GHz}$. The input matching is designed for the minimum NF. The inter-stage and output matching networks are conjugately matched for the maximum gain. Fig. 2 shows the threestage schematic of the proposed LNA. All the input, output, and inter-stage matching networks are implemented using thin-film microstrip lines (TFMSs) [3].

\section{B. RF ESD Protection Designs}

ESD protection is usually needed at all pads to prevent IC damage caused by ESD stress. The phenomenon of ESD stress happens in short time duration with a current spike of several amperes [4]. To enhance the voltage and current robustness level of the ESD protection, a large protection device is required. However, the large parasitic capacitances associated with the large protection devices will affect the RF performance significantly. Apparently, the conventional RF ESD protection can not be applied to MMW circuit design. In recent years, there are a few approaches to improve the core circuit performance with RF ESD protection [5]-[7].

Distributed circuit architecture was used to achieve broadband impedance matching with the ESD protection device along a transmission line, as shown in Fig. 3 [5], [6]. Assume the total capacitance of the ESD device to be $C_{\mathrm{ESD}}$, the length of the

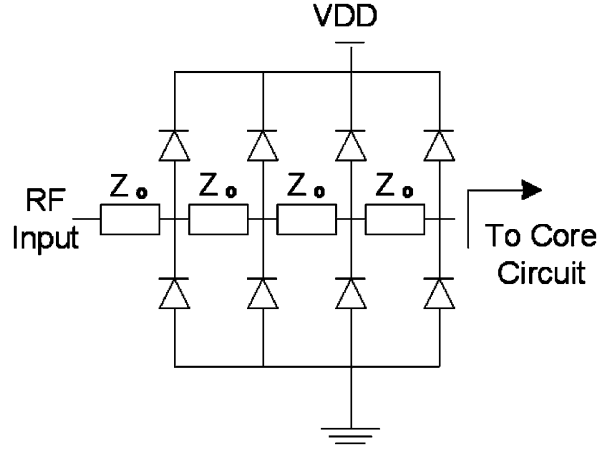

Fig. 3. Distributed RF ESD protection.

transmission line should be chosen to match to a $50-\Omega$ characteristic impedance according the following equation:

$$
Z_{o}=\sqrt{\frac{L_{\text {line }}}{C_{\text {line }}+C_{\mathrm{ESD}}}}=50 \Omega
$$

where $L_{\text {line }}$ and $C_{\text {line }}$ represent the inductance and parasitic capacitance of the transmission line. Due to good impedance matching, distributed ESD structures can achieve broadband performance. In low microwave frequencies, to achieve 50- $\Omega$ input impedance will require a large area, even to millimeter scale. On the other hand, as the operation frequency goes higher to the MMW frequency, the high insertion loss becomes the major concern.

Fig. 4(a) shows the T-coil network structure [5]. A T-coil network consists of two coupled inductors $L_{1}$ and $L_{2}$ with a coupling coefficient $k$, and a bridge capacitor $C_{B}$. At low frequencies, the input node will short to the resistor $R_{t}$ via $L_{1}$ and $L_{2}$, while at high frequencies, $C_{B}$ acts as another short path to $R_{t}$. This circuit presents a resistive impedance looking into the input port. As the $50-\Omega$ characteristic impedance is matched, it will lead to an ultra-wideband performance if the following conditions hold:

$$
Z_{\text {in }}=R_{t}=50 \Omega
$$




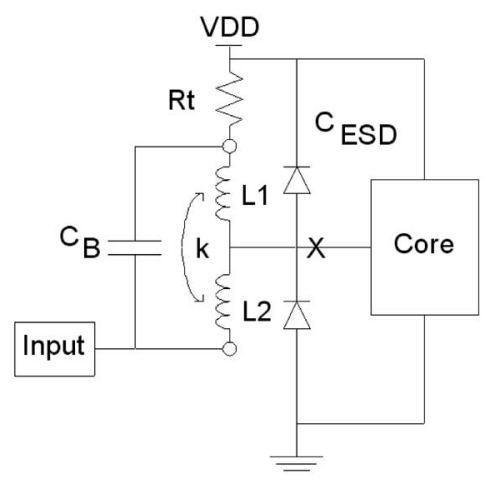

(a)

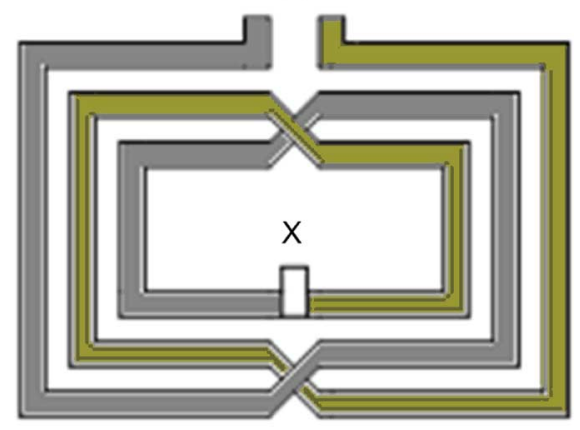

(b)

Fig. 4. (a) ESD protection of the T-coil network structure. (b) Layout of the $\mathrm{T}$-coil transformer.

$$
\begin{aligned}
L_{1} & =L_{2}=\frac{C_{T} R_{t}^{2}}{3} \\
C_{B} & =\frac{C_{T}}{12} \\
k & =\frac{1}{2}
\end{aligned}
$$

where $C_{T}$ is the total capacitance for the ESD device and the input capacitance of the core circuit. The physical layout of the T-coil transformer is shown in Fig. 4(b). However, the T-coil network will suffer a great loss at MMW frequencies. Besides, an inductor with a high- $Q$ factor and high resonant frequency is difficult to implement. Thus, the T-coil network would not be suitable in $V$-band RF ESD design.

Fig. 5 shows the configuration of the $L C$-tank [7] RF ESD protection. The $L C$-tank is designed to resonant at the operation frequency. The value of the inductance and capacitance can be obtained from the resonating frequency as the equation:

$$
f_{o}=\frac{1}{2 \pi \sqrt{L C}} .
$$

During the RF circuit operates, the ESD device is blocked by the $L C$-tank, which presents a high impedance. The RF performance then will not be degraded due to the parasitic capacitance of ESD devices. On the other hand, with consideration on the ESD robustness, the resonant capacitance can be replaced by an ESD device, which provides another short path for discharging. Unfortunately, due to the limitation of the resonant frequency, the $L C$-tank can only cover a narrow band. At MMW frequencies, the $L C$-tank structure will suffer a high insertion loss. For

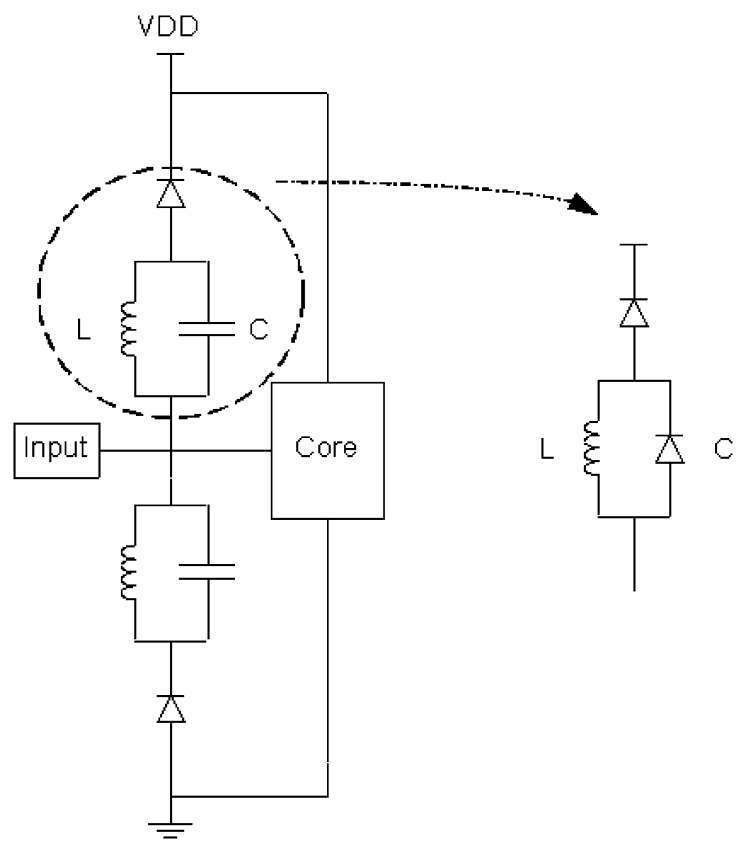

Fig. 5. $L C$-tank RF ESD protection.

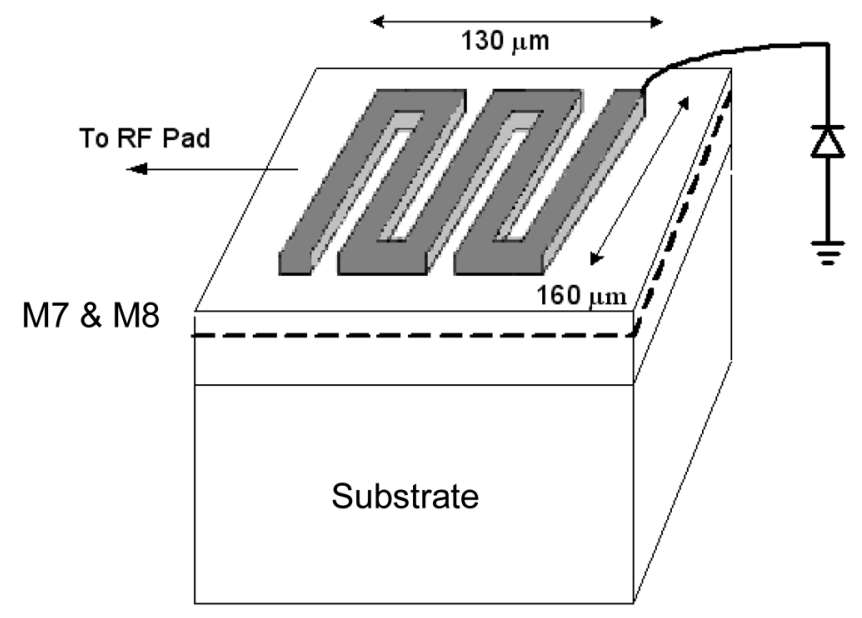

Fig. 6. 3-D view of the proposed RF ESD protection.

example, the simulation shows that an $L C$-tank designed to resonate at $60 \mathrm{GHz}$ has more than $3.5-\mathrm{dB}$ insertion loss.

We can conclude from the above discussions that beside ESD robustness, wideband and high-frequency response are required in MMW CMOS RF ESD protection circuits.

Fig. 6 illustrates the 3-D view of the proposed RF ESD protection. It consists of a silicon controlled rectifier (SCR) [8] and a meander inductor. The SCR is realized based on parasitic n-p-n/p-n-p inherent to CMOS devices, and can provide a low-impedance path to discharge the ESD current. The meander inductor is fabricated with metal 7 and metal 8, but without metal 1 as the ground plane, since the inductor with ground plane will have higher parasitic capacitance, and thus, limit the inductance. At $60 \mathrm{GHz}$, an SCR (total gatewidth $=500 \mu \mathrm{m}$ ) with a parasitic capacitance $0.35 \mathrm{pF}$ is close to a short circuit. The ESD circuit can then be considered as a one-port network of a meander inductor short to ground, as stated in [9]. Fig. 7(a) 


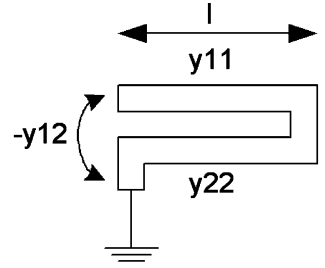

(a)

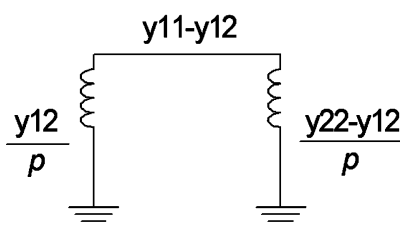

(b)

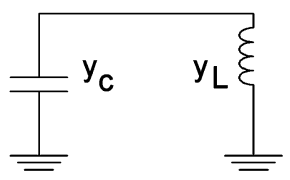

(c)

Fig. 7. (a) One-port short-circuited meander-line. (b) Equivalent circuit model. (c) Equivalent circuit model with Richard's transformation.

shows the onefold meander inductor short to ground, with equivalent model of $y$-parameters. $1 / y_{11}$ and $1 / y_{22}$ represents the inductances for each line of length $\ell$, and $y_{12}$ is used to model the coupling effects. According to the analysis in [9], the meander inductor can be equivalent to the model, as shown in Fig. 7(b), where $p$ represents Richard's variable $(p=j \tan \beta \ell)$ [10]. According to Richard's theorem, it can be transformed to an $L C$ network shown in Fig. 7(c) by the following equations [9]:

$$
\begin{aligned}
& y_{L}=y_{12}+\frac{\left(y_{11}-y_{12}\right)\left(y_{22}-y_{12}\right)}{y_{11}+y_{22}-2 y_{12}} \\
& y_{c}=\frac{\left(y_{11}-y_{12}\right)^{2}}{y_{11}+y_{22}-2 y_{12}} .
\end{aligned}
$$

To avoid the degradation of RF performance, the impedance isolation is used to shield the ESD device and the core circuit. In order to transform a short circuit to open, the sixfold meander inductor is used with a compact size of $0.16 \times 0.13 \mathrm{~mm}^{2}$, as shown in Fig. 8(a).

The sixfold meander inductor can be treated as a six-stage $L C$ network, as shown in the equivalent circuit of Fig. 8(b), where $L_{m}$ represents the inductance of the metal line, and $C_{m}$ is the coupling capacitance. $C_{\text {sub }}$ and $R_{\text {sub }}$ are the parasitic capacitance and resistance due to the lossy substrate. If $C_{m}, C_{\mathrm{sub}}$, and $R_{\text {sub }}$ are negligible, the meander inductor could be considered as a series inductor. Consequently, as looking into the input port, the meander inductor with the ESD device presents a high input impedance, and the circuit will retain its RF performance. The equivalent circuit model parameters of the meander inductor can be extracted by fitting $S$-parameters obtained from EM simulation (Sonnet Software). In the equivalent circuit model, we obtained $L_{m}=0.075 \mathrm{nH}, C_{m}=4 \mathrm{fF}, C_{\mathrm{sub}}=8 \mathrm{fF}$, and $R_{\mathrm{sub}}=600 \Omega$. It is observed that the effects of $C_{m}, C_{\mathrm{sub}}$,



(a)

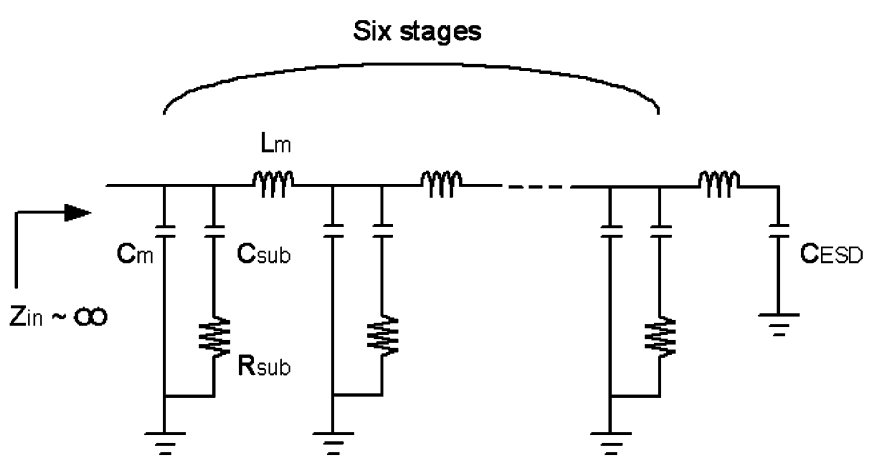

(b)

Fig. 8. (a) Sixfold meander inductor. (b) Equivalent circuit.

and $R_{\text {sub }}$ are not significant and can be neglected. Therefore, the meander inductor behaves as a series inductor, and the input impedance is about $j 6 \omega L_{m}$.

The simulated inductance of the meander inductor is shown in Fig. 9(a). We can see that the inductor features a very wideband resonant frequency. From 50 to $75 \mathrm{GHz}$, the input impedance of the meander inductor connected with SCR shows very high impedance. The circuit schematic of the RF ESD-protected LNA is shown in Fig. 10. Since the ESD network is shunt with the core circuit and exhibits high impedance, the overall performance of the core circuit will not be affected so much. Fig. 11 presents the simulated $S$-parameters of the shunt connection of the meander inductor, which features a bandpass filter performance. It is observed that the insertion losses are less than $2 \mathrm{~dB}$, and the return losses are better than $10 \mathrm{~dB}$ from 50 to $75 \mathrm{GHz}$. Therefore, the ESD protection can provide broadband impedance isolation on the RF path and low impedance on the discharging path. The wideband performance can indeed be used for the full $V$-band $(50-75 \mathrm{GHz})$ applications. Fig. 12 shows a chip photograph of the proposed 60-GHz LNA with RF ESD protection. The die size of the chip is $0.94 \times 0.76 \mathrm{~mm}^{2}$, including all testing pads and dummy metals, while the area for the ESD protection is $0.18 \times 0.4 \mathrm{~mm}^{2}$. In order to verify the ESD function, the LNA without RF ESD protection is also designed for comparison. Fig. 13 illustrates the chip photograph of the LNA without the ESD protection with a die size of $0.72 \times 0.67 \mathrm{~mm}^{2}$. Dummy layers are kept away from the signals so as not to influence the RF signals. 


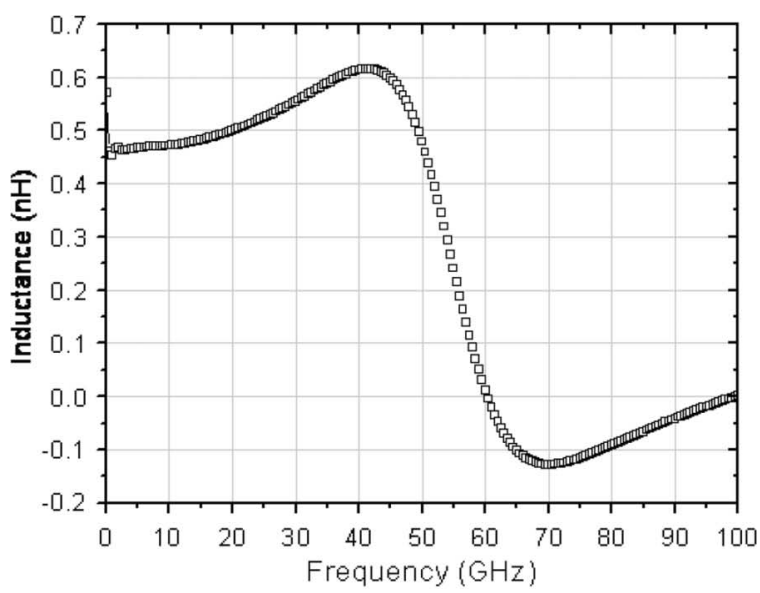

(a)

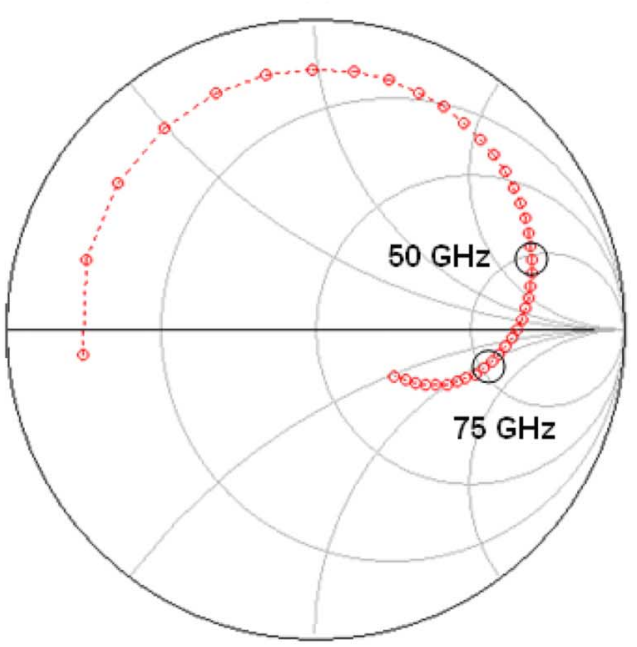

(b)

Fig. 9. (a) Inductance. (b) Input impedance of the meander inductor.

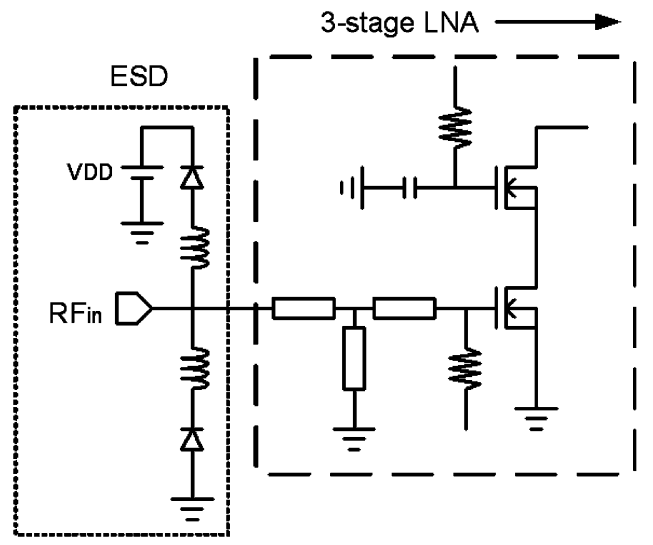

Fig. 10. Proposed ESD-protected LNA.

\section{EXPERIMENTAL RESULTS}

\section{A. LNA Measurements and Comparison}

The LNA chip was measured via on-wafer probing using Agilent's HP8510 $V$-band test set. Both the LNAs with and without ESD protection were fabricated on the same wafer and tested for comparison. Fig. 14 plots the measured small-signal gain and input/output return losses from 45 to $75 \mathrm{GHz}$ at 2.4-V drain

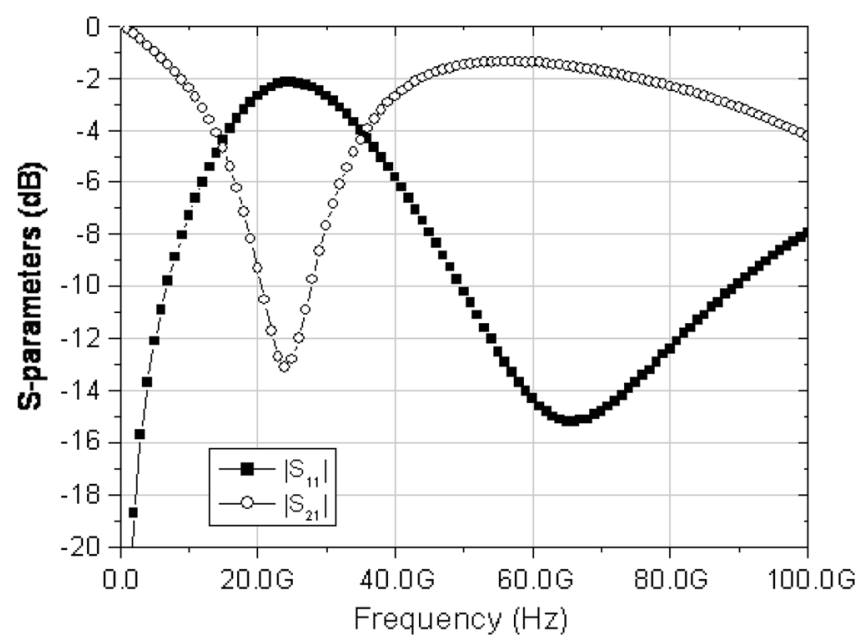

Fig. 11. Simulated $S$-parameters of the ESD protection structure.



Fig. 12. Chip photograph of ESD-protected LNA with die size of $0.94 \times 0.76 \mathrm{~mm}^{2}$.

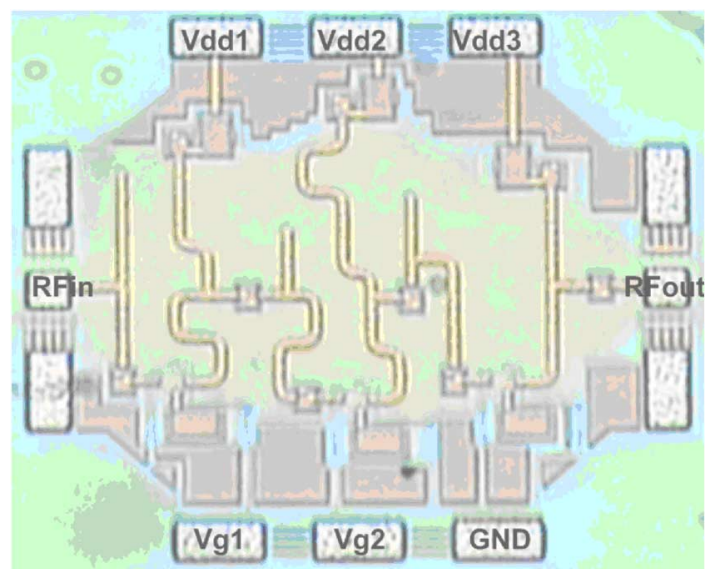

Fig. 13. Chip photograph of unprotected LNA with die size of $0.72 \times$ $0.67 \mathrm{~mm}^{2}$.

bias with total current of $27 \mathrm{~mA}$. At this bias condition, the drain-source voltages $\left(V_{\mathrm{DS}}\right)$ of each transistor in the cascode configuration are $1.2 \mathrm{~V}$. The hollow line represents $S$-parameters of the LNA without the ESD protection, and the solid line represents that with RF ESD protection. The measured smallsignal gain of the LNA without ESD protection is higher than 


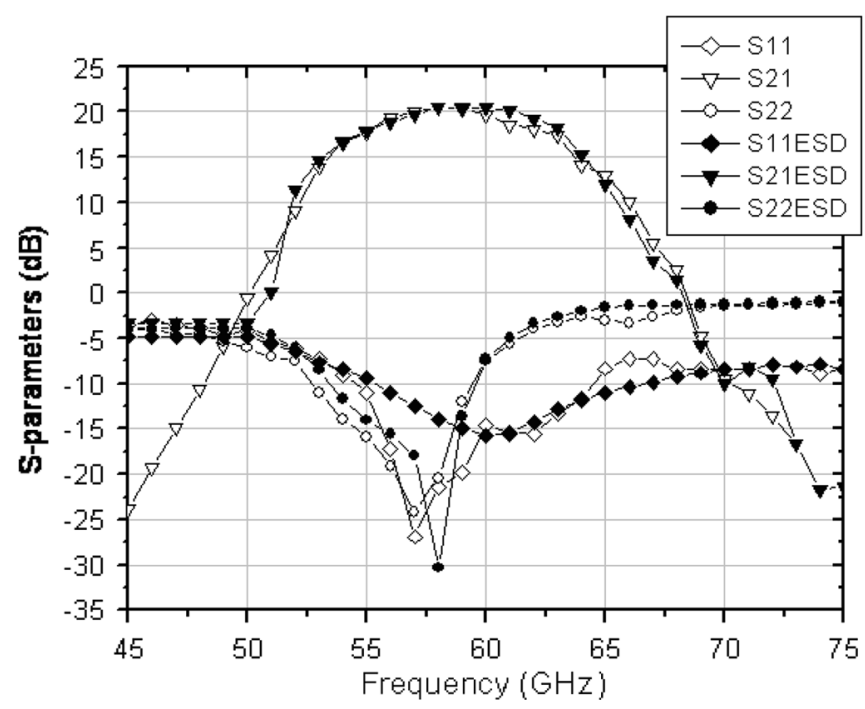

Fig. 14. Measured small-signal $S$-parameters of the protected and unprotected LNAs.

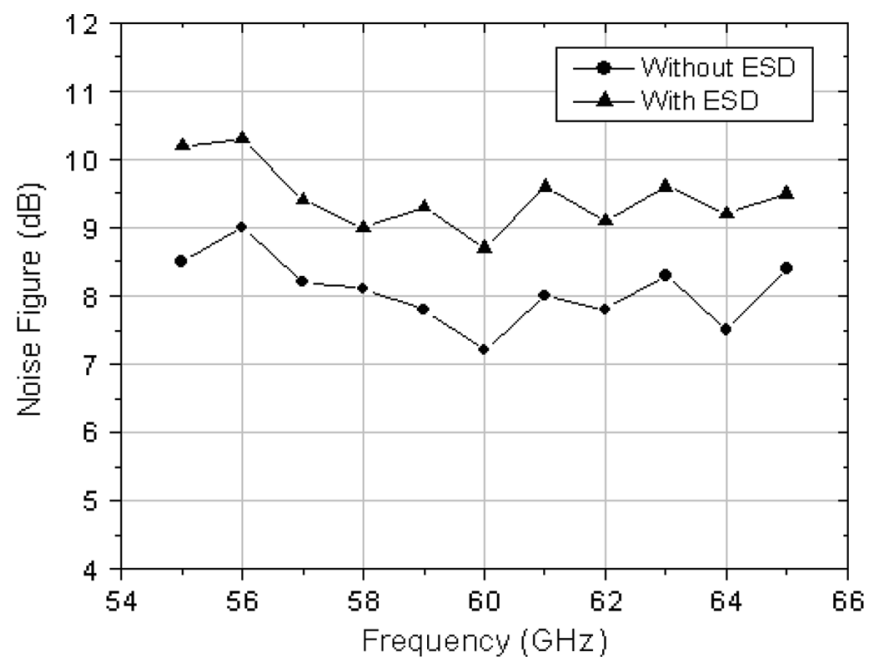

Fig. 15. Measured NFs of the protected and unprotected LNAs.

$17 \mathrm{~dB}$ from 55 to $62 \mathrm{GHz}$ with a 20.2-dB peak gain at $58 \mathrm{GHz}$. The input and output return losses are better than 10 and $5 \mathrm{~dB}$, respectively. With ESD protection, the circuit has almost the same small-signal gain and return losses at $58 \mathrm{GHz}$. The measured NF at the same bias condition is shown in Fig. 15. The line with circles is the measured NF of the LNA without ESD protection, and the line with triangles is that with ESD protection. The LNA without ESD protection exhibits an average NF of $8 \mathrm{~dB}$ with a minimum of $7.2 \mathrm{~dB}$ at $60 \mathrm{GHz}$. The ESD-protected LNA shows an average NF of $9.3 \mathrm{~dB}$ with a minimum of $8.6 \mathrm{~dB}$. The variation in the NF between that with and without ESD protection is about $1.5 \mathrm{~dB}$, which is partly contributed by the parasitic resistance of the SCR and the meander inductor. On the other hand, the first stage of the ESD-protected LNA connected with the input port via a $180-\mu \mathrm{m}$ TFMS line results in a little mismatch of noise, and this also contributes some access NF. Under the 2.4-V/27-mA bias condition, the input 1-dB compression point $\left(P_{1 \mathrm{~dB}}\right), \mathrm{IP} 3$, and output saturation power are $-20,-12$, and $5.2 \mathrm{dBm}$, respectively, which are all close to those of the

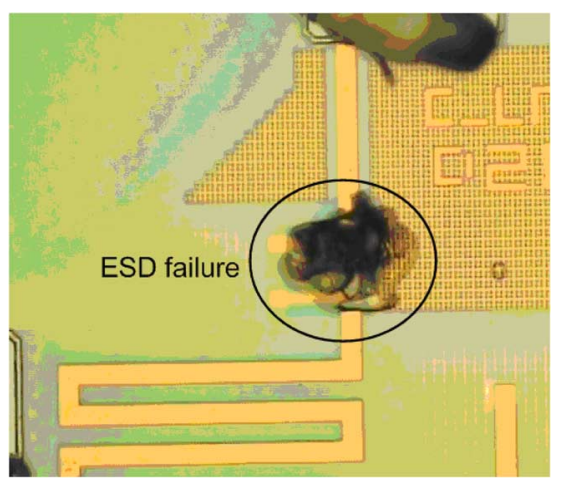

Fig. 16. ESD failure of the diode under more than $6.5-\mathrm{kV}$ stressing.

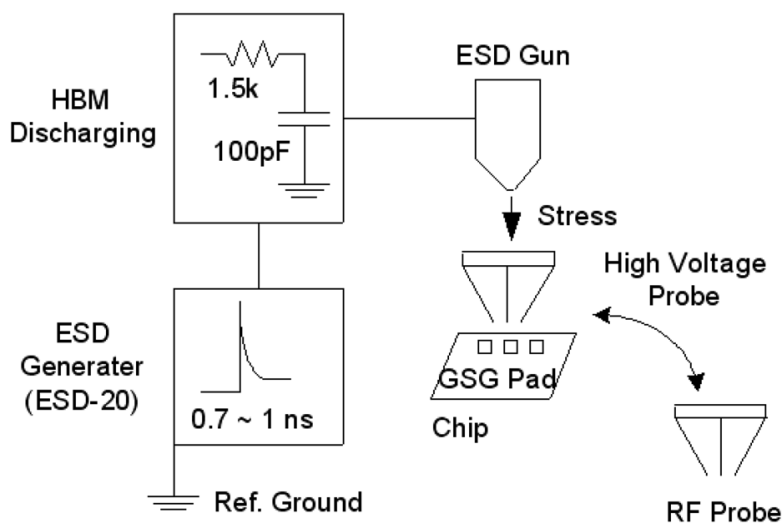

Fig. 17. ESD measurement setup.

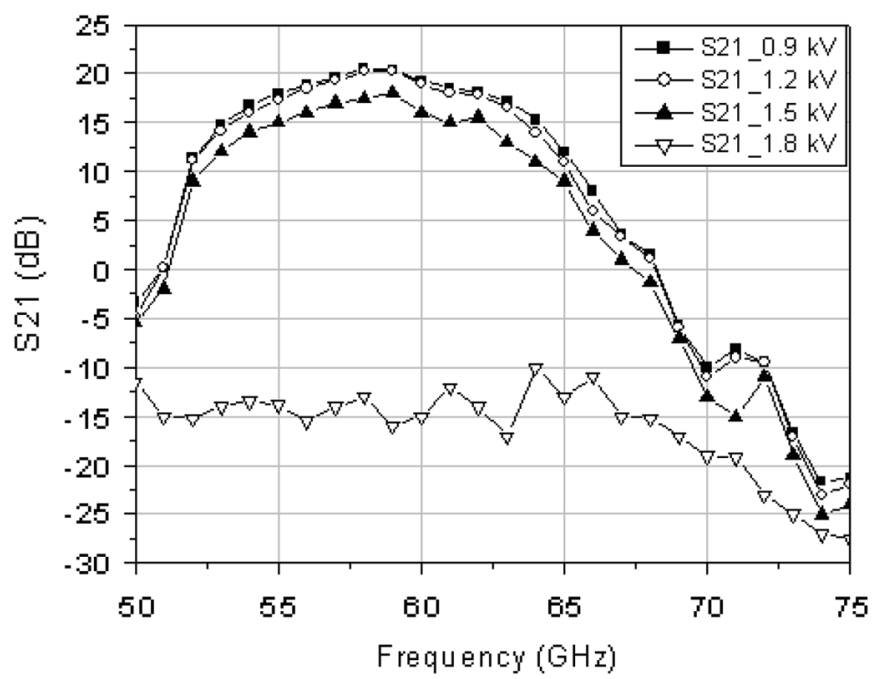

Fig. 18. Measured $S_{21}$ under various ESD stressing.

LNA without ESD protection. The total dc power consumption of these two LNAs are both $65 \mathrm{~mW}$.

\section{B. ESD Test}

For the voltage robustness test of the ESD protection, an ESD generator (ESD-20) supplied by Suzhou Everich is used. The ESD generator can provide $0.1-20-\mathrm{kV}$ positive and negative voltage pulses. The attenuated testing output waveform presents that a high voltage pulse of 1-ns duration can be generated. In the mean while, a discharge mode of HBM is utilized for 
TABLE I

COMPARISON OF PREVIOUSLY REPORTED RF ESD-PROTECTED LNAS

\begin{tabular}{|c|c|c|c|c|c|c|c|c|}
\hline Technology & $\begin{array}{c}\text { Freq. } \\
(\mathrm{GHz})\end{array}$ & $\begin{array}{c}\text { Gain } \\
(\mathrm{dB})\end{array}$ & $\begin{array}{c}\text { NF with } \\
\text { ESD }(\mathrm{dB})\end{array}$ & $\begin{array}{c}\text { NF Variation } \\
(\text { with/without ESD }) \\
(\mathrm{dB})\end{array}$ & $\begin{array}{c}\text { Chip Size } \\
\left(\mathrm{mm}^{2}\right)\end{array}$ & $\begin{array}{c}\text { ESD Area } \\
\left(\mathrm{mm}^{2}\right)\end{array}$ & $\begin{array}{c}\text { HBM Level } \\
(\mathrm{kV})\end{array}$ & Ref./Year \\
\hline $\begin{array}{c}0.25-\mu \mathrm{m} \\
\text { CMOS }\end{array}$ & 2.7 & N/A & 0.8 & 0.2 & N/A & N/A & 2 & {$[7] / 2003$} \\
\hline $\begin{array}{c}0.35-\mu \mathrm{m} \\
\text { BiCMOS }\end{array}$ & $3-5$ & 12 & 2.1 & N/A & 1.134 & 0.02 & 6.5 & {$[11] / 2006$} \\
\hline $\begin{array}{c}90-\mathrm{nm} \\
\text { CMOS }\end{array}$ & 2.4 & 21.9 & 3.2 & 1 & 1.44 & 0.08 & 4 & {$[12] / 2006$} \\
\hline $\begin{array}{c}0.15-\mu \mathrm{m} \\
\text { pHEMT }\end{array}$ & $11.7-12.75$ & 24.5 & 1.24 & N/A & N/A & N/A & 4.4 & {$[13] / 2003$} \\
\hline $\begin{array}{c}0.18-\mu \mathrm{m} \\
\text { BiCMOS }\end{array}$ & 7 & 19 & 4 & 1 & N/A & N/A & 1.5 & {$[14] / 2007$} \\
\hline $\begin{array}{c}90-\mathrm{nm} \\
\text { CMOS }\end{array}$ & $5-6$ & 13.3 & 2.9 & 0.5 & 0.94 & 0.08 & 2 & {$[15] / 2005$} \\
\hline $\begin{array}{c}\mathbf{0 . 1 3 - \mu m} \\
\text { CMOS }\end{array}$ & $\mathbf{5 5 - 6 2}$ & $\mathbf{2 0 . 4}$ & $\mathbf{8 . 6}$ & $\mathbf{1 . 5}$ & $\mathbf{0 . 7 1 5}$ & $\mathbf{0 . 0 7}$ & $\mathbf{6 . 5 / 1 . 5}$ & This Work \\
\hline
\end{tabular}

ESD stress. The equivalent circuit consists of a 100-pF capacitor and a $1.5-\mathrm{k} \Omega$ resistor to simulate the zapping of human body. ESD-20 generates the discharge voltage pulse to the ESD gun, and then the RF pad is stressed via the bond wire. Under $6.5-\mathrm{kV}$ testing, the ESD failure and damage can be observed, as shown in Fig. 16. However, at MMW frequencies, the small device of the core circuit is very sensitive to ESD stress. To ensure the function of the core circuit, a high-voltage robustness probe is utilized to test the RF pad. The $S$-parameters and dc characteristics can then be measured again. The measurement setup for ESD stress is shown in Fig. 17. The ESD voltage pulse directly stresses on the probe connected with RF pad via HBM discharge mode, and then the RF probe is exchanged to measure the $S$-parameters again. Before testing, the pulse voltage has been calibrated to the probe tip. The real output ESD pulse is from 0.35 to $19.8 \mathrm{kV}$. The small-signal gain versus frequency under various ESD stressing is shown in Fig. 18. Under 0.9- and 1.2-kV stresses, the LNA still retains small signal gain of higher than $17 \mathrm{~dB}$. After $1.5-\mathrm{kV}$ testing, the gain of LNA decreases about $2-3 \mathrm{~dB}$, but the LNA is still functional from 55 to $62 \mathrm{GHz}$. As the stressing voltage increases to $1.8 \mathrm{kV}$, a sharp gain drop is observed. In the meanwhile, the LNA exhibits a failure dc characteristic and the transistor is damaged. Consequently, the LNA is determined to be failed.

Table I summaries the previously reported LNAs with RF ESD protection and compares them with this design. It is observed that our LNA achieves a respectable RF performance and ESD robustness with a compact size. This is also the first ESDprotected CMOS LNA in the MMW regime and has the highest operation frequency among the reported LNAs with ESD protection reported to date.

\section{CONCLUSION}

A 60-GHz LNA RF ESD protection in TSMC commercial MS/RF purpose $0.13-\mu \mathrm{m}$ CMOS technology has been demonstrated in this paper. The chip achieves a small-signal gain of $20.4 \mathrm{~dB}$ and an NF of $8.7 \mathrm{~dB}$, which exhibited 0.2-dB difference in gain and $1.5 \mathrm{~dB}$ in noise compared with the LNA without ESD protection. Under $2.4-\mathrm{V}$ drain bias, the LNA consumed
65-mW dc power dissipation. The measured input $P_{1 \mathrm{~dB}}$ and IP3 at $60 \mathrm{GHz}$ are -20 and $-12 \mathrm{dBm}$, respectively. The chip size including the proposed ESD protection is $0.94 \times 0.76 \mathrm{~mm}^{2}$. The diodes failed under more than $6.5-\mathrm{kV}$ HBM testing and the core circuit can be still functional under $1.5-\mathrm{kV}$ testing.

\section{ACKNOWLEDGMENT}

The authors would like to thank C.-M. Lo, C.-S. Lin, and T.-P. Wang, all with National Taiwan University, Taipei, Taiwan, for their helpful suggestions.

The chip was fabricated by Taiwan Semiconductor (TSMC) through the Chip Implementation Center of Taiwan.

\section{REFERENCES}

[1] M.-D. Ker and K.-H. Lin, "Design on ESD protection scheme for IC with power-down-mode operation," IEEE J. Solid-State Circuits, vol. 39, no. 8, pp. 1378-1382, Aug. 2004.

[2] M.-D. Ker, W.-Y. Chen, and K.-C. Hsu, "Design on power rail ESD clamp circuit for $3.3-\mathrm{V}$ I/O interface by using only $1-\mathrm{V} / 2.5-\mathrm{V}$ lowvoltage devices in130-nm CMOS process," IEEE Trans. Circuits Syst., vol. 53, no. 10, pp. 2187-2193, Oct. 2006.

[3] C.-M. Lo, C.-S. Lin, and H. Wang, "A miniature $V$-band 3-stage cascode LNA in $0.13 \mu \mathrm{m}$ CMOS," in Int. Solid-State Circuits Conf. Tech. Dig., Feb. 2006, pp. 402-403.

[4] H. Feng et al., "A full-monolithic LNA in $0.18 \mu \mathrm{m}$ SiGe: Performance variation due to ESD protection," in Proc. 7th Int. Solid-State Integr. Circuits Technol. Conf., Oct. 18-21, , pp. 1226-1229.

[5] S. Galal and B. Razavi, "Broadband ESD protection circuits in CMOS technology," IEEE J. Solid-State Circuits, vol. 38, no. 12, pp. 2334-2340, Dec. 2003.

[6] M.-D. Ker and B.-J. Kuo, "Decreasing-size distributed ESD protection scheme for broadband RF circuits," IEEE Trans. Microw. Theory Tech., vol. 53, no. 2, pp. 582-589, Feb. 2006.

[7] M.-D. Ker, C.-I. Chou, and C.-M. Lee, "A novel $L C$-tank ESD protection design for gigahertz RF circuits," in Radio Frequency Integr. Circuits Symp. Dig., Jun. 2003, pp. 115-118.

[8] A. Chtterjee and T. Polgreen, "A low-voltage trigging SCR for on-chip ESD protection at output and input pads," IEEE Electron Device Lett., vol. 12, no. 1, pp. 21-22, Jan. 1991.

[9] R. Sato, "A design method for meander-line networks using equivalent circuit transformations," IEEE Trans. Microw. Theory Tech., vol. MTT-19, no. 5, pp. 431-442, May 1971.

[10] P. I. Richards, "Resistor-transmission-line circuits," Proc. IRE, vol. 36, no. 2, pp. 217-220, Feb. 1948.

[11] M. Liu et al., "A 6.5-kV ESD-protected 3-5-GHz ultra- wideband BiCMOS low-noise amplifier using interstage gain roll-off compensation," IEEE Trans. Microw. Theory Tech., vol. 54, no. 4, pp. 1698-1706, Apr. 2006. 
[12] C.-P. Chang et al., "A high gain and supply voltage LNA for direct conversion application with 4-kV HBM ESD protection in 90-nm RF CMOS," IEEE Microw. Wireless Compon. Lett., vol. 16, no. 11, pp. 612-614, Nov. 2006.

[13] C.-K. Park et al., " $K u$-band low noise amplifier with short- stub ESD protection," in Radio Freq. Integr. Circuits Symp. Dig., Jun. 2003, pp. 671-674.

[14] K. Bhatia, S. Hyvonen, and E. Rosebaum, "A compact, ESD -protected, SiGe BiCMOS LNA for ultra-wideband applications," IEEE J. SolidState Circuits, vol. 5, no. 8, pp. 1121-1130, May 2007.

[15] D. Linten et al., "A $5 \mathrm{GHz}$ fully integrated ESD protected low noise amplifier in 90-nm RF CMOS," IEEE J. Solid-State Circuits, vol. 40, no. 7, pp. 1434-1442, Jul. 2005.

[16] T. Yao et al., "Algorithmic design of CMOS LNAs and PAs for 60-GHz radio," IEEE J. Solid-State Circuits, vol. 42, no. 5, pp. 1044-1057, May 2007.

[17] S. Emami, C. H. Doan, A. M. Niknejad, and R. W. Brodersen, "A highly integrated $60 \mathrm{GHz}$ CMOS front-end receiver," in Int. Solid-State Circuits Conf. Tech. Dig., Feb. 2007, pp. 190-191.

[18] B. Rasavi, "A 60-GHz CMOS receiver front-end," IEEE J. Solid-State Circuits, vol. 41, no. 1, pp. 17-22, Jan. 2006.

[19] B. Rasavi, "A mm-wave heterodyne receiver with on-chip LO and divider," in Int. Solid-State Circuits Conf. Tech. Dig., Feb. 2007, pp. 188-189.

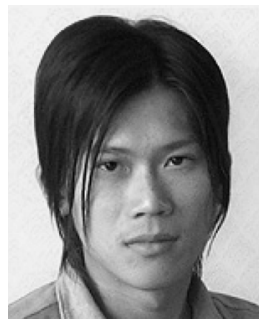

Bo-Jr Huang (S'08) was born in Tainan, Taiwan, on April 19, 1982. He received the B.S. degree in electrical engineering from National Cheng Kung University, Tainan, Taiwan, in 2005, and is currently working toward the Ph.D. degree at National Taiwan University, Taipei, Taiwan.

His research interests include microwave and MMW IC designs.

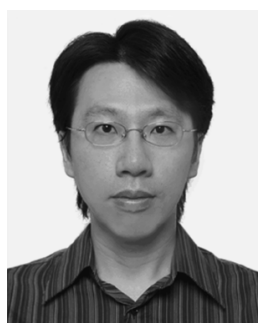

Chi-Hsueh Wang (S'02-M'05) was born in Kaohsiung, Taiwan, in 1976. He received the B.S. degree in electrical engineering from National Cheng Kung University, Tainan, Taiwan, in 1997, and the Ph.D. degree from National Taiwan University, Taipei, Taiwan, in 2003.

His research interests include the design and analysis of microwave and MMW circuits and computational electromagnetics.



Chung-Chun Chen ( $\left.\mathrm{S}^{\prime} 03\right)$ was born in Taipei, Taiwan, in 1979. He received the M.S. degree in communication engineering from National Taiwan University, Taipei, Taiwan, in 2004, and is currently working toward the Ph.D. degree in electronics engineering from National Taiwan University.

His current research interests focus on MMW ICt designs for high-speed communication systems.

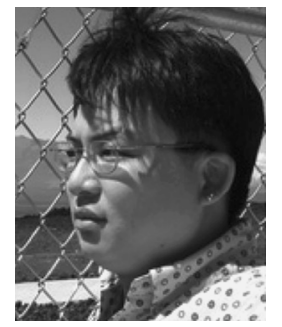

Ming-Fong Lei (S'00-M'08) was born in Taipei, Taiwan, on December 20, 1980. He received the B.S. degree in electrical engineering and Ph.D. degree in communication engineering from National Taiwan University, Taipei, Taiwan, in 2002 and 2007, respectively.

His research interests include the design and analysis of microwave and MMW circuits, microwave device modeling, and filters.

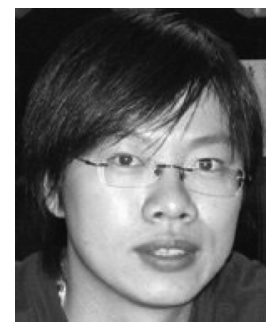

Pin-Cheng Huang was born in Kaohsiung, Taiwan, on May 18, 1982. He received the B.S. degree in electrical engineering from National Cheng Kung University, Taiwan, in 2004, and is currently working toward the Ph.D. degree at National Taiwan University, Taipei.

His research interests include the design and analysis of microwave and MMW amplifiers, mixers, and oscillators.

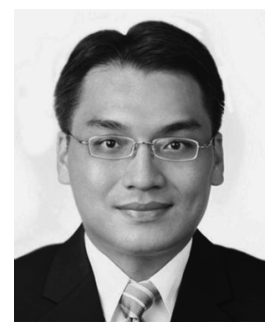

Kun-You Lin (S'00-M'04) was born in Taipei, Taiwan, in 1975. He received the B.S. degree in communication engineering from National Chiao Tung University, Hsinchu, Taiwan, in 1998, and the $\mathrm{Ph} . \mathrm{D}$. degree in communication engineering from National Taiwan University, Taipei, Taiwan, in 2003.

From August 2003 to March 2005, he was a Post-Doctoral Research Fellow with the Graduate Institute of Communication Engineering, National Taiwan University. From May 2005 to July 2006, he was an Advanced Engineer with the Sunplus Technology Company Ltd., Hsin-Chu, Taiwan. In July 2006, he joined the faculty of the Department of Electrical Engineering and Graduate Institute of Communication Engineering, National Taiwan University, as an Assistant Professor. His research interests include the design and analysis of microwave/RF circuits.

Dr. Lin is a member of Phi Tau Phi.

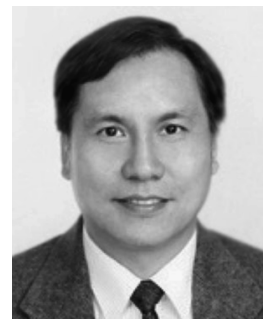

Huei Wang (S'83-M'87-SM'95-F'06) was born in Tainan, Taiwan, on March 9, 1958. He received the B.S. degree in electrical engineering from National Taiwan University, Taipei, Taiwan, in 1980, and the M.S. and Ph.D. degrees in electrical engineering from Michigan State University, East Lansing, in 1984 and 1987, respectively.

During his graduate study, he was engaged in research on theoretical and numerical analysis of EM radiation and scattering problems. He was also involved in the development of microwave remote detecting/sensing systems. In 1987, he joined the Electronic Systems and Technology Division, TRW Inc. He has been an MTS and Staff Engineer responsible for MMIC modeling of computer-aided design (CAD) tools, MMIC testing evaluation, and design and became the Senior Section Manager of the MMW Sensor Product Section, RF Product Center. In 1993, he visited the Institute of Electronics, National Chiao-Tung University, Hsinchu, Taiwan, to teach MMIC-related topics. In 1994, he returned to TRW Inc. In February 1998, he joined the faculty of the Department of Electrical Engineering, National Taiwan University, as a Professor. He is currently the Director of the Graduate Institute of Communication Engineering, National Taiwan University.

Dr. Wang is a member of Phi Kappa Phi and Tau Beta Pi. He was the recipient of the Distinguished Research Award of National Science Council (2003-2006). In 2005, he was elected as the first Richard M. Hong Endowed Chair Professor of National Taiwan University. He has been appointed an IEEE Distinguished Microwave Lecturer for the 2007-2009 term. 